\title{
Conditions for nonmonotonic vortex interaction in two-band superconductors
}

\author{
A. Chaves, ${ }^{1,2}$ L. Komendová, ${ }^{1}$ M. V. Milošević, ${ }^{1}$ J. S. Andrade Jr., ${ }^{2}$ G. A. Farias, ${ }^{2}$ and F. M. Peeters ${ }^{1,2, *}$ \\ ${ }^{1}$ Departement Fysica, Universiteit Antwerpen, Groenenborgerlaan 171, B-2020 Antwerpen, Belgium \\ ${ }^{2}$ Departamento de Física, Universidade Federal do Ceará, 60455-900 Fortaleza, Ceará, Brazil
}

(Received 11 May 2011; published 30 June 2011)

\begin{abstract}
We describe a semianalytic approach to the two-band Ginzburg-Landau theory, which predicts the behavior of vortices in two-band superconductors. We show that the character of the short-range vortex-vortex interaction is determined by the sign of the normal domain-superconductor interface energy, in analogy with the conventional differentiation between type I and type II superconductors. However, we also show that the long-range interaction is determined by a modified Ginzburg-Landau parameter $\kappa^{*}$, different from the standard $\kappa$ of a bulk superconductor. This opens the possibility for nonmonotonic vortex-vortex interaction, which is temperature dependent, and can be further tuned by alterations of the material on the microscopic scale.
\end{abstract}

DOI: 10.1103/PhysRevB.83.214523

PACS number(s): 74.25.Dw, 74.70.Ad, 74.70.Xa

\section{INTRODUCTION}

Multigap superconductivity arises when the gap amplitudes on different sheets of the Fermi surface are radically disparate, for example, due to different dimensionality of the bands for the usual phonon-mediated pairing, as is the case in $\mathrm{MgB}_{2},{ }^{1}$ or due to the repulsive pairing interaction, as it appears to be the case in recently discovered iron-pnictides. ${ }^{2,3}$ The other examples of multigap materials include $\mathrm{OsB}_{2}$, iron silicides such as $\mathrm{Lu}_{2} \mathrm{Fe}_{3} \mathrm{Si}_{5}$, chalcogenides $\left(\mathrm{NbSe}_{2}\right)$, but also the conventional superconductors such as $\mathrm{Pb}$ when reduced to nanoscale. ${ }^{4}$

In a strong magnetic field all superconducting condensates form normal-metal voids, as an intermediate state before superconductivity is fully destroyed. These normal domains tend to merge in type I superconductors in order to minimize their positive surface energy, whereas in type II superconductors they have negative surface energy and split into quantized vortices. However, in 2005 Babaev and Speight predicted the so-called semi-Meissner state in two-band superconductors, ${ }^{5}$ the state with localized regions of high and low vortex densities, arising from short-range repulsive while long-range attractive vortex-vortex interaction. This vortex behavior was recently visualized by Moshchalkov et al., ${ }^{6}$ in the form of stripes and clusters of vortices in a single-crystal $\mathrm{MgB}_{2}$. Such vortex configurations stemming from the long-range attractive vortex behavior (see also Ref. 7 for review) are clearly very important in the field of superconductivity, but they also present a bridge between solid-state physics and soft condensed matter, where systems with competing interactions are of abiding interest. ${ }^{8}$

To date, the matter of competing vortex interactions in two-band superconductors has not been conclusively settled although recent years saw a surge of activities in this field. The original prediction in Ref. 5 concerns only the case when one band is type I and the other type II, although it is unclear how different types of behavior between bands in $k$-space (not real space) can be discerned. Reference 9 demonstrated such vortex behavior in systems where just one band is fully superconducting, and the other superconducts only due to direct coupling. Dao et al. found different types of possible vortex-vortex interactions and several resulting exciting vortex configurations, but did not provide a universal criterion to a priori determine the type of vortex interaction. ${ }^{10}$ Finally, some authors expressed skepticism to nonmonotonic vortex interaction; Geyer et al. showed that the normal metal/two-gap superconductor surface energy close to $T_{c}$ depends just on a single Ginzburg-Landau (GL) parameter $\kappa$, and thus only either repulsive (type II) or attractive (type I) vortex-vortex interaction is possible. ${ }^{11}$ This point was later reenforced by Kogan and Schmalian. ${ }^{12}$

\section{METHODS AND DERIVATIONS}

\section{A. The Ginzburg-Landau formalism for two-band superconductors}

In this paper we derive criteria for the appearance of nonmonotonic interaction of vortices in two-gap systems described by the standard GL model. Our analysis is based on the two-band GL theory, but with correct microscopic parameters obtained either from theoretical band structure calculations or by fitting the experimental penetration depth or specific heat data by the so-called $\gamma$ model. ${ }^{13}$ We begin from the GL energy functional, which comprises single-band contributions from both condensates, the coupling term, and the energy of the magnetic field in and around the sample:

$$
\begin{aligned}
\mathcal{F}= & \sum_{j=1,2} \alpha_{j}\left|\Psi_{j}\right|^{2}+\frac{1}{2} \beta_{j}\left|\Psi_{j}\right|^{4}+\frac{1}{2 m_{j}}\left|\left(\frac{\hbar}{i} \nabla-\frac{2 e}{c} \mathbf{A}\right) \Psi_{j}\right|^{2} \\
& -\Gamma\left(\Psi_{1}^{*} \Psi_{2}+\Psi_{1} \Psi_{2}^{*}\right)+\frac{(\mathbf{h}-\mathbf{H})^{2}}{8 \pi} .
\end{aligned}
$$

Here the two Cooper-pair condensates are described by the order parameters $\Psi_{1}$ and $\Psi_{2}, \mathbf{H}$ is the applied magnetic field, and $\mathbf{h}$ the net one. The Josephson coupling term provides the "minimal coupling," well described in literature. The temperature enters the energy expression through $\alpha_{j=1,2}$, linearly dependent on the temperature term $\tau=\ln T_{c} / T \approx$ $1-T / T_{c} \cdot{ }^{14}$ The expansion leading to the GL theory is strictly valid only in the immediate vicinity of $T_{c}$, but we use this theory at somewhat lower temperatures as well, arguing that GL theory qualitatively well describes important physics away from $T_{c}$ (as was demonstrated at many prior instances). Finally, it was shown in Ref. 12, that standard two-band GL theory contains incomplete terms that estimate $\psi$ with precision to 
$\tau^{3 / 2}$. The authors reduce the theory by eliminating latter terms, which results in a single coherence length for both order parameters of a two-band superconductor. This is, however, not a correct physical picture at low temperatures, ${ }^{15}$ and two coherence lengths for the two-band superconductors can be recovered even in the GL domain in the extended model of Ref. 16. Unfortunately, the latter model is presented in the absence of magnetic field. To be able to capture all the essential physics, at least qualitatively, we base our study on the compromise standard GL model. Note, however, that our further explained semianalytic approach can be applied to any improved form of the energy functional for two-band superconductors.

We next calculate the vortex-vortex interaction in a similar fashion to Ref. 5 but within a correct microscopic framework. The parameters in Eq. (1) can then be expressed as $\alpha_{j}=-N(0) n_{j} \chi_{j}=-N(0) n_{j}\left(\tau-S_{j} / n_{j} \eta\right), \beta_{j}=$ $N(0) n_{j} / W^{2}, \quad m_{j}=3 W^{2} / N(0) n_{j} v_{j}^{2}$, and $\Gamma=N(0) \lambda_{12} / \eta$, where

$$
\Lambda=\left|\begin{array}{cc}
\lambda_{11} & \lambda_{12} \\
\lambda_{21}=\lambda_{12} & \lambda_{22}
\end{array}\right|
$$

is the coupling matrix with determinant $\eta ; N(0)$ denotes total and $n_{j} N(0)$ partial density of states, $v_{j}$ are the Fermi velocities in the two bands, and $W^{2}=8 \pi^{2} T_{c}^{2} / 7 \zeta(3)$. For details on constants $S_{j}$, we refer to Ref. 12. This allows us to technically define the coherence lengths $\xi_{j}=\frac{\hbar v_{j}}{\sqrt{6} W}$ and penetration depths $\lambda_{j}=\sqrt{\frac{3 c^{2}}{16 \pi N(0) e^{2} n_{j} v_{j}^{2}}}$, as well as the GL parameters $\kappa_{j}=\lambda_{j} / \xi_{j}$ of the two condensates, as if they were independent. These are, however, just parameters of the model, and are related only indirectly with the resulting penetration depth and the healing lengths of the two order parameters in the two-band material. Notice also that $\alpha_{1}$ and $\alpha_{2}$ change sign at different temperatures. In particular, close to $T_{c}$ both $\alpha_{j}$ are positive but the coupled system is still superconducting. Such situation is already different from the one studied in Ref. 9, where at least one $\alpha_{j}$ was negative. The GL equations minimize the functional from Eq. (1) and read (in dimensionless form)

$$
\begin{gathered}
(-i \nabla-\mathbf{A})^{2} \Psi_{1}-\left(\chi_{1}-\left|\Psi_{1}\right|^{2}\right) \Psi_{1}-\gamma \Psi_{2}=0 \\
(-i \nabla-\mathbf{A})^{2} \Psi_{2}-\alpha\left(\chi_{2}-\left|\Psi_{2}\right|^{2}\right) \Psi_{2}-\frac{\gamma \kappa_{2}^{2}}{\kappa_{1}^{2} \alpha} \Psi_{1}=0 \\
-\triangle \mathbf{A}=\kappa_{1}^{-2} j_{1}+\alpha \kappa_{2}^{-2} j_{2},
\end{gathered}
$$

where $j_{j}=\mathfrak{R}\left[\Psi_{j}^{*}(-i \nabla-\mathbf{A}) \Psi_{j}\right], \quad \alpha=\left(v_{1} / v_{2}\right)^{2}, \quad \gamma=$ $\Gamma / n_{1} N(0)$, both order parameters are scaled to $W$, distances to $\xi_{1}$, and vector potential to $h c / 4 e \pi \xi_{1}$.

\section{B. Long-range vortex interaction}

In what follows, we demonstrate the method to determine the asymptotic long-range interaction of vortices, before going into fine details at short vortex-vortex distances. In cylindrical coordinates, considering the ansatz for one circular symmetric vortex $\Psi_{j}=e^{i \theta} f_{j}(r)$, and substituting the gauge
$\vec{A}=a(r) \hat{\theta} / r$, we rewrite GL Eqs. (2a)-(2c) as

$$
\frac{d^{2} f_{1}}{d r^{2}}+\frac{1}{r} \frac{d f_{1}}{d r}-\frac{(a-1)^{2}}{r^{2}} f_{1}+\left(\chi_{1}-f_{1}^{2}\right) f_{1}+\gamma f_{2}=0
$$

$\frac{d^{2} f_{2}}{d r^{2}}+\frac{1}{r} \frac{d f_{2}}{d r}-\frac{(a-1)^{2}}{r^{2}} f_{2}+\alpha\left(\chi_{2}-f_{2}^{2}\right) f_{2}+\frac{\gamma}{\alpha} \frac{\kappa_{2}^{2}}{\kappa_{1}^{2}} f_{1}=0$,

and

$$
\frac{d^{2} a}{d r^{2}}-\frac{1}{r} \frac{d a}{d r}-(a-1)\left(\frac{f_{1}^{2}}{\kappa_{1}^{2}}+\alpha \frac{f_{2}^{2}}{\kappa_{2}^{2}}\right)=0 .
$$

For $r \rightarrow \infty, a$ converges to 1 and $f_{j}$ to a constant $a_{j}$. The limit $r \rightarrow \infty$ leads to the set of nonlinear coupled equations for $a_{j}$ :

$$
\begin{gathered}
\left(\chi_{1}-a_{1}^{2}\right) a_{1}+\gamma a_{2}=0, \\
\alpha\left(\chi_{2}-a_{2}^{2}\right) a_{2}+\frac{\gamma}{\alpha} \frac{\kappa_{2}^{2}}{\kappa_{1}^{2}} a_{1}=0 .
\end{gathered}
$$

These can be decoupled by defining the ratio $\rho=a_{1} / a_{2}$, which then obeys the fourth-order equation

$$
\frac{\gamma}{\alpha^{2}} \frac{\kappa_{2}^{2}}{\kappa_{1}^{2}} \rho^{4}+\chi_{2} \rho^{3}-\chi_{1} \rho-\gamma=0 .
$$

Such an equation has a laborious analytical solution known as Ferrari's method, which is not presented here, but can be found in Ref. 17. From Eq. (4), one obtains the dependence of the constants $a_{j}$ on the ratio $\rho$ as

$$
\begin{gathered}
a_{1}=\sqrt{\frac{\gamma}{\rho}+\chi_{1}}, \\
a_{2}=\sqrt{\frac{\gamma}{\alpha^{2}} \frac{\kappa_{2}^{2}}{\kappa_{1}^{2}} \rho+\chi_{2} .}
\end{gathered}
$$

In order to eliminate high order terms for large distances, we must use auxiliary functions that approach zero as $r \rightarrow \infty$, namely, $Q(r)=a(r)-1$ and $\sigma_{j}(r)=f_{j}(r)-a_{j}$. Keeping only first-order terms in these functions, Eqs. (3) become

$$
\begin{gathered}
\frac{d^{2} \sigma_{1}}{d r^{2}}+\frac{1}{r} \frac{d \sigma_{1}}{d r}+\left(\chi_{1}-3 a_{1}^{2}\right) \sigma_{1}+\gamma \sigma_{2}=0, \\
\frac{d^{2} \sigma_{2}}{d r^{2}}+\frac{1}{r} \frac{d \sigma_{2}}{d r}+\alpha\left(\chi_{2}-3 a_{2}^{2}\right) \sigma_{2}+\frac{\gamma}{\alpha} \frac{\kappa_{2}^{2}}{\kappa_{1}^{2}} \sigma_{1}=0,
\end{gathered}
$$

and

$$
\frac{d^{2}}{d r^{2}}\left(\frac{Q}{r}\right)+\frac{1}{r} \frac{d}{d r}\left(\frac{Q}{r}\right)-\left(\frac{\xi_{1}^{2}}{\lambda^{2}}-\frac{1}{r^{2}}\right)\left(\frac{Q}{r}\right)=0,
$$

where we defined $\lambda^{-2}=\left(a_{1} / \lambda_{1}\right)^{2}+\left(a_{2} / \lambda_{2}\right)^{2}$. The solution of Eq. (7c) is the modified Bessel function $Q(r)=$ $\delta_{3} r K_{1}\left(r \xi_{1} / \lambda\right)$. Similarly, if $\gamma=0$, Eqs. (7a) and (7b) are decoupled and easily identified as modified Bessel equations, whose solutions are $\sigma_{1}(r)=\eta_{1} K_{0}\left(\sqrt{2 \chi_{1}} r\right)$ and $\sigma_{2}(r)=$ $\eta_{2} K_{0}\left(\sqrt{2 \alpha \chi_{2}} r\right)$. On the other hand, if $\gamma \neq 0$, the equations for $\sigma_{j}$ are still coupled and, in order to decouple them, one must define the operator $\hat{L}_{2}=\nabla^{2}+\alpha\left(\chi_{2}-3 a_{2}^{2}\right)$, so that $\hat{L}_{2} \sigma_{2}=-\left(\gamma \kappa_{2}^{2} / \alpha \kappa_{1}^{2}\right) \sigma_{1}$, and apply it on Eq. (7a), obtaining

$$
\nabla^{2} \nabla^{2} \sigma_{1}+C_{1} \nabla^{2} \sigma_{1}+C_{2} \sigma_{1}=0
$$


Here $C_{1}=\left(\chi_{1}-3 a_{1}^{2}\right)+\alpha\left(\chi_{2}-3 a_{2}^{2}\right)$ and $C_{2}=\alpha\left(\chi_{2}-\right.$ $\left.3 a_{2}^{2}\right)\left(\chi_{1}-3 a_{1}^{2}\right)-\gamma^{2} \kappa_{2}^{2} / \alpha \kappa_{1}^{2}$. The operator $\nabla^{2}$ for axially symmetric solutions has eigenfunctions given by Bessel functions $J_{0}(\beta r)$ and $Y_{0}(\beta r)$, with eigenvalue $-\beta^{2}$, or modified Bessel functions $I_{0}(\beta r)$ and $K_{0}(\beta r)$, with eigenvalue $\beta^{2}$. From these four eigenfunctions, only the latter satisfies the condition that $\sigma_{j}$ must decay monotonically with $r$. Substituting $\nabla^{2} K_{0}(\beta r)=$ $\beta^{2} K_{0}(\beta r)$ in Eq. (8), one obtains

$$
\beta^{4}+C_{1} \beta^{2}+C_{2}=0
$$

and

$$
\begin{aligned}
& \sigma_{1}(r)=\delta_{1} \cos (\omega) K_{0}\left(\beta_{-} r\right)-\delta_{2} \sin (\omega) K_{0}\left(\beta_{+} r\right), \\
& \sigma_{2}(r)=\delta_{1} \sin (\omega) K_{0}\left(\beta_{-} r\right)+\delta_{2} \cos (\omega) K_{0}\left(\beta_{+} r\right),
\end{aligned}
$$

where

$$
\beta_{ \pm}=\sqrt{\frac{-C_{1} \pm \sqrt{C_{1}^{2}-4 C_{2}}}{2}} .
$$

Notice that in Eqs. (10), each $\sigma_{j}$ must contain the Bessel functions for both $\beta_{ \pm}$, in a combination that is conveniently written in the form of a mixing angle $\omega .{ }^{9}$ In the $\gamma \rightarrow 0$ limit, one has $\beta_{-} \rightarrow \sqrt{2 \chi_{1}}$ and $\beta_{+} \rightarrow \sqrt{2 \alpha \chi_{2}}$. Moreover, substituting Eqs. (10) in the differential equation (7a), one obtains

$$
\tan (\omega)=\frac{\gamma}{\beta_{+}^{2}+\left(\chi_{1}-3 a_{1}^{2}\right)}
$$

so that $\gamma \rightarrow 0$ leads to $\omega \rightarrow 0$ and, consequently, to $\sigma_{1}(r) \rightarrow$ $\eta_{1} K_{0}\left(\sqrt{2 \chi_{1}} r\right)$ and $\sigma_{2}(r) \rightarrow \eta_{2} K_{0}\left(\sqrt{2 \alpha \chi_{2}} r\right)$, as expected.

The parameters $\delta_{k}\left(\eta_{k}\right)$ in the expressions for $Q(r), \sigma_{1}(r)$, and $\sigma_{2}(r)$ are unknown real constants that can only be determined by fitting numerical solutions for Eqs. (3) in analogy to what is done in Ref. 18.

Having the asymptotic form of the order parameters and the vector potential, we now follow the standard procedure ${ }^{19}$ for finding the vortex-vortex interaction in the $r \rightarrow \infty$ limit, obtaining

$$
E_{2 B}(\mathrm{r})=\delta_{3}^{2} K_{0}\left(\frac{\mathrm{r}}{\lambda}\right)-\delta_{1}^{2} K_{0}\left(\frac{\beta_{-} \mathrm{r}}{\xi_{1}}\right)-\delta_{2}^{2} K_{0}\left(\frac{\beta_{+} \mathrm{r}}{\xi_{1}}\right),
$$

where the units are now explicitly shown. Here we list the consequences of the above asymptotics. (i) Comparing Eq. (12) to the one-band case, ${ }^{20}$ where

$$
E_{1 B}(\mathrm{r})=\delta_{4}^{2} K_{0}\left(\mathrm{r} / \lambda_{1 B}\right)-\delta_{5}^{2} K_{0}\left(\sqrt{2} \mathrm{r} / \xi_{1 B}\right),
$$

shows that the length scale $\lambda^{-2}=\left(a_{1} / \lambda_{1}\right)^{2}+\left(a_{2} / \lambda_{2}\right)^{2}$ is playing the role of an effective penetration depth for the two-band superconductor in accordance with Eq. (60) in Ref. 21 , contrary to $\lambda^{-2}=\left(1 / \lambda_{1}\right)^{2}+\left(1 / \lambda_{2}\right)^{2}$ used in Refs. 5 and 22 , which holds only in the (unrealistic) absence of coupling. (ii) The parameters $\delta_{k}$ are, in general, different from each other, but can be calculated exactly in the Bogomol'nyi point for the two-band system as $\delta_{1}^{2}=\delta_{2}^{2}=2 \delta_{3}^{2}$. For $\gamma=0$, the choice of $\xi_{1}=\xi_{2}=1$ and $\kappa_{1}=\kappa_{2}=1$ in the two-band case is thus analogous to the Bogomol'nyi point $\kappa_{1 B}=1 / \sqrt{2}$ for the single-band case and, accordingly, the long-range interaction must vanish (and change sign for $\kappa_{1}=\kappa_{2}<1$ ). This directly illustrates that coupling of two (nominally) type II condensates may lead to a type I behavior of the coupled system. (iii) In Eq. (13) for single-band superconductors, it is clear that if $\kappa_{1 B}=\lambda_{1 B} / \xi_{1 B}>1 / \sqrt{2}(<1 / \sqrt{2})$, the interaction potential $E_{1 B}(\mathrm{r})$ will be repulsive (attractive). For two-band superconductors, Eq. (12) shows that the relevant parameters are $\kappa_{ \pm}^{*}=\frac{\beta_{ \pm} \lambda}{\sqrt{2} \xi_{1}}$, rather than the nominal GL parameters $\kappa_{j}$ for each condensate. If either $\kappa_{+}^{*}$ or $\kappa_{-}^{*}$ is below $1 / \sqrt{2}$, the long-range vortex interaction is attractive (type I like). Equations (4) and (10c) provide simple means to evaluate this condition. (iv) In the presence of coupling, the long-range behavior of both $\sigma_{j}$ depends exponentially on the smallest of $\beta_{-}$and $\beta_{+}$. Therefore, in the coupled case, we can define not only a single penetration depth for both bands, but also the order parameters for both condensates exhibit the same decay at large distances, which implies a joint coherence length $\xi^{*}=\xi_{1} / \min \left(\beta_{+}, \beta_{-}\right)$.

\section{Surface energy and the short-range vortex interaction}

The analysis in the previous subsection brings us to the discussion of the real criterion for the attractive/repulsive nature of the vortex interaction. In the single-band case, the changing sign of the normal domain-superconductor surface energy $E_{S}$ at the Bogomol'nyi point is a correct criterion. However, in the two-band case and for large vortex-vortex distance, the Bogomol'nyi point is determined by a single valued $\kappa^{*}=\min \left(\kappa_{+}^{*}, \kappa_{-}^{*}\right)=1 / \sqrt{2}$, which is not necessarily where the surface energy of the normal domain (vortex) changes sign.

The sign of the energy of the interfaces between normalmetal domains and the superconductor determines whether merging of those domains is energetically favorable or not (i.e., if the superconductor is type I or type II). In the case of vortices, the smallest possible normal domains, the negative vortex-superconductor surface energy therefore means that the vortices should repel (at least at short distances) in order to avoid the formation of a giant vortex. We here show how to calculate the normal-superconducting interface energy and by that predict the type of the short-range vortex-vortex interaction.

We follow a similar approach to that of Ref. 22, but we take into account the Josephson coupling and the temperature dependence of the GL parameters, within a correct microscopical framework. Namely, we consider the interface between normal and superconducting region as the $y z$ plane at $x=0$ and calculate the surface energy $E_{S}$ using the one-dimensional GL functional at the thermodynamic critical field $H_{c c}$, which reads

$$
\begin{aligned}
E_{S}= & \int_{-\infty}^{\infty} d x\left\{2\left(\Psi_{1}^{\prime 2}+A^{2} \Psi_{1}^{2}\right)+\left(2 \chi_{1}-\Psi_{1}^{2}\right) \Psi_{1}^{2}\right. \\
& +\alpha \frac{\kappa_{1}^{2}}{\kappa_{2}^{2}}\left[2\left(\Psi_{2}^{\prime 2}+A^{2} \Psi_{2}^{2}\right)+\alpha\left(2 \chi_{2}-\Psi_{2}^{2}\right) \Psi_{2}^{2}\right] \\
& \left.-2 \gamma \Psi_{1} \Psi_{2}+\left(H_{c c}-\sqrt{2} \kappa_{1} A^{\prime}\right)^{2}\right\},
\end{aligned}
$$

where the gauge potential is chosen as $\vec{A}=[0, A(x), 0]$ and $\Psi_{j=1,2}$ are taken real. The thermodynamic critical field of the 
coupled system $H_{c c}$ is obtained from the condition that the GL functional in Eq. (1) converges to zero for $H=H_{c c}$, leading to

$H_{c c}^{2}=H_{c(1)}^{2} a_{1}^{2}\left(2 \chi_{1}-a_{1}^{2}\right)+H_{c(2)}^{2} a_{2}^{2}\left(2 \chi_{2}-a_{2}^{2}\right)+4 \gamma H_{c(1)}^{2} a_{1} a_{2}$,

where the formally defined thermodynamic critical field of each condensate is $H_{c(j)}=h c /\left(4 e \sqrt{2} \pi \lambda_{j} \xi_{j}\right)=$ $2 W \sqrt{\pi N(0) n_{j}}$. We then find $\Psi_{j}$ and $A$ that minimize $E_{S}$ by numerically solving the set of Euler-Lagrange equations for the functional in Eq. (14), which are exactly the one-dimensional versions of Eqs. (2a)-(2c):

$$
\begin{gathered}
\Psi_{1}^{\prime \prime}=\frac{A^{2}}{2} \Psi_{1}-\left(\chi_{1}-\Psi_{1}^{2}\right) \Psi_{1}-\gamma \Psi_{2}, \\
\Psi_{2}^{\prime \prime}=\frac{A^{2}}{2} \Psi_{2}-\alpha\left(\chi_{2}-\Psi_{2}^{2}\right) \Psi_{2}-\frac{\gamma \kappa_{2}^{2}}{\alpha \kappa_{1}^{2}} \Psi_{1}, \\
A^{\prime \prime}=\left(\frac{\Psi_{1}^{2}}{\kappa_{1}^{2}}+\alpha \frac{\Psi_{2}^{2}}{\kappa_{2}^{2}}\right) A .
\end{gathered}
$$

The boundary conditions in the normal state $(x \rightarrow-\infty)$ and deep in the superconducting state $(x \rightarrow \infty)$ are $\psi_{j}(x \rightarrow$ $-\infty)=0, A^{\prime}(x \rightarrow-\infty)=1, \psi_{j}^{\prime}(x \rightarrow \infty)=0$, and $A^{\prime}(x \rightarrow$ $\infty)=0$.

\section{Constrained GL equations for fixed vortices}

We supplement our argumentation by numerically obtained vortex-vortex interaction potentials (in a similar fashion as in Ref. 23). Since the problem of two vortices does not have circular symmetry, we now consider the fixed-vortex ansatz in Cartesian coordinates $\Psi_{j}=e^{i n_{1} \theta_{1}} e^{i n_{2} \theta_{2}} f_{j}(x, y)$, describing two fixed vortices with winding numbers $n_{1}$ and $n_{2}$, where $e^{i n_{k} \theta_{k}}$ is written in Cartesian coordinates as

$$
e^{i n_{k} \theta_{k}}=\left(\frac{x_{k}+i y_{k}}{x_{k}-i y_{k}}\right)^{n_{k} / 2}
$$

and $\vec{r}_{k}=\left(x_{k}, y_{k}, 0\right)$ is the in-plane position vector with origin at the center of the vortex $k$. For the case of two vortices separated by a distance $d$, we take $\vec{r}_{1}=(x-d / 2, y, 0)$ and $\vec{r}_{2}=(x+$ $d / 2, y, 0)$. With this ansatz, the Euler-Lagrange equations for the energy functional in Eq. (1) read (see also Ref. 23)

$$
\begin{aligned}
& \nabla^{2} f_{1}-\left[\bar{X}^{2}+\bar{Y}^{2}+2\left(A_{x} \bar{Y}-A_{y} \bar{X}\right)+\vec{A}^{2}\right] f_{1} \\
& \quad+\left(\chi_{1}-f_{1}^{2}\right) f_{1}+\gamma f_{2}=0 \\
& \nabla^{2} f_{2}-\left[\bar{X}^{2}+\bar{Y}^{2}+2\left(A_{x} \bar{Y}-A_{y} \bar{X}\right)+\vec{A}^{2}\right] f_{2} \\
& \quad+\alpha\left(\chi_{2}-f_{2}^{2}\right) f_{2}+\frac{\gamma \kappa_{2}^{2}}{\alpha \kappa_{1}^{2}} f_{1}=0
\end{aligned}
$$

and

$$
\vec{\nabla} \times \vec{\nabla} \times \vec{A}=-\left[\vec{A}-\frac{n_{1} \hat{\theta}_{1}}{r_{1}}-\frac{n_{2} \hat{\theta}_{2}}{r_{2}}\right]\left(\frac{f_{1}^{2}}{\kappa_{1}^{2}}+\alpha \frac{f_{2}^{2}}{\kappa_{2}^{2}}\right),
$$

where

$$
\bar{X}=\frac{n_{1} x_{1}}{r_{1}^{2}}+\frac{n_{2} x_{2}}{r_{2}^{2}}, \quad \bar{Y}=\frac{n_{1} y_{1}}{r_{1}^{2}}+\frac{n_{2} y_{2}}{r_{2}^{2}},
$$

and the angular unit vectors around each vortex are written as $\widehat{\theta_{k}}=\left(-y_{k} / r_{k}, x_{k} / r_{k}, 0\right)$.
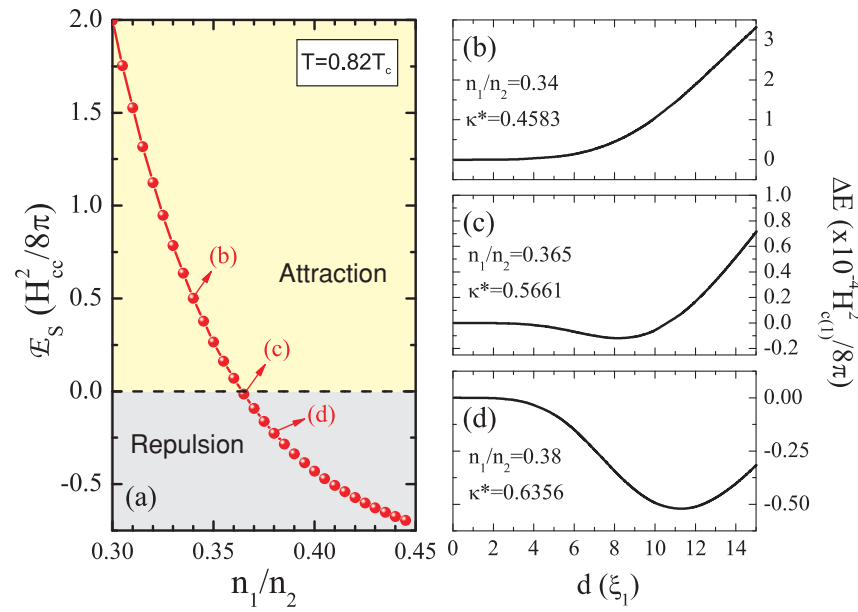

FIG. 1. (Color online) The normal domain-superconductor surface energy $E_{S}$ as a function of the ratio of the density of states in the two bands (a) and the corresponding vortex-vortex interaction energies (b)-(d) for indicated parameters. The short-range interaction force changes sign when the surface energy changes sign.

Equations (18a)-(18c) are thus the GL equations for the two fixed vortices, and we solve them numerically by a relaxation method. The obtained order parameter and vector potential are then substituted back in the energy functional, yielding the energy $E(d)$ for the vortex pair at distance $d$. Repeating this procedure for different vortex-vortex separation, we obtain the interaction potential $\Delta E=E(d)-E(0)$ between vortices in the two-gap superconductor, as shown in Figs. 1(b)-1(d).

\section{RESULTS AND DISCUSSION}

We now apply the techniques described in the previous sections to calculate (i) the asymptotic long-range GL parameter $\kappa^{*}$, (ii) the normal domain-superconductor surface energy $E_{S}$, and (iii) the full vortex-vortex potential (using the constrained GL equations (18a)-(18c)).

As a first example, Fig. 1 shows the surface energy $E_{S}$ and the numerically obtained vortex-vortex interaction potentials for a set of parameters corresponding (arguably) to $\mathrm{MgB}_{2}$ : $\kappa_{1}=3.71$ and $\xi_{1} / \xi_{2}=v_{1} / v_{2}=0.255$ are taken from Ref. 6 , the coupling matrix is obtained from Ref. 24 , the temperature is fixed at $T=0.82 T_{c}$, while we vary the density of states in the two bands. We note that in all considered cases $\kappa^{*}<1 / \sqrt{2}$ and the long-range interaction is always attractive, whereas short-range interaction changes to repulsive exactly when the surface energy $E_{S}$ changes sign with increasing $n_{1} / n_{2}$. To conclude, the long-range vortex-vortex interaction is determined by $\kappa^{*}$ with respect to $1 / \sqrt{2}$, while the short-range behavior is determined by the sign of the surface energy $E_{S}$. This also proves insufficient the initial premise in Ref. 6 that if the system has $\lambda / \xi_{1}>1 / \sqrt{2}$ and $\lambda / \xi_{2}<1 / \sqrt{2}$, the vortex interaction should be long-range attractive and short-range repulsive. The actual behavior is far more complex and can be exactly determined as explained above.

Recent calculations have shown that as $T \rightarrow T_{c}$, only type I or type II vortex behavior can be observed. ${ }^{11}$ Indeed, by analyzing $\kappa^{*}$ and the sign of $E_{S}$ at $T \rightarrow T_{c}$ as explained above, 


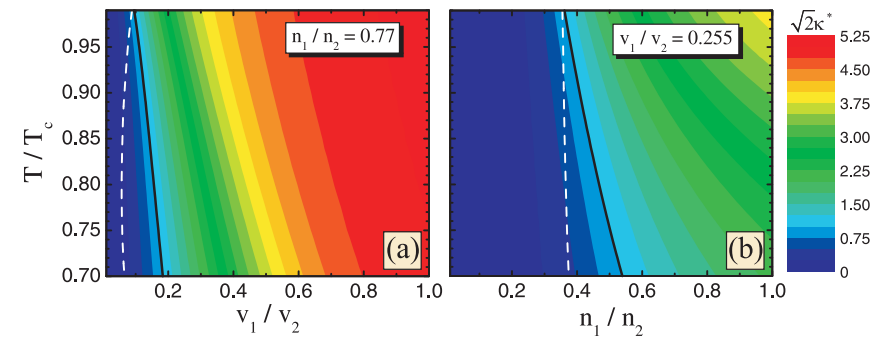

FIG. 2. (Color online) The long-range interaction phase diagram for a $\mathrm{MgB}_{2}$ crystal, at different temperatures and for varied values of the ratio between (a) the Fermi velocities and (b) the partial density of states of the two bands. In each panel, the black line separates the regions of long-range attraction and long-range repulsion (left to right). The white lines indicate where $E_{S}$ changes sign and the short-range interaction changes from attractive to repulsive (left to right).

we always found the same type of interaction in either longor short-range limit. However, for $T$ immediately below $T_{c}$ the sign change of $E_{S}$ and the transition from $\kappa^{*}>1 / \sqrt{2}$ to $\kappa^{*}<1 / \sqrt{2}$ occur for different sets of parameters, opening up the parameter space for observation of the nonmonotonic vortex interaction. This is shown in Fig. 2, where we plot $\kappa^{*}$ as a function of temperature and also the ratios between the Fermi velocities [Fig. 2(a)] and the partial density of states of each condensate [Fig. 2(b)]. The black line in Fig. 2 denotes $\kappa^{*}=1 / \sqrt{2}$ and the white line indicates where $E_{S}=0$. At $T=T_{c}$ these lines coincide, in agreement with Ref. 11, but as $T$ decreases, the lines separate, bordering the region where the system exhibits short-range repulsion $\left(E_{S}<0\right)$ and longrange attraction $\left(\kappa^{*}<1 / \sqrt{2}\right)$, that is, nonmonotonic vortex interaction. This finding further creates a new possibility of tuning the magnetic interactions in two-band superconductors by changing temperature. For example, for the parameters of $\mathrm{MgB}_{2}$ given in Ref. 6 [Fig. 2(a) for $v_{1} / v_{2}=0.255$ ], we find that nonmonotonic vortex interactions occur only for $T \lesssim$ $0.49 T_{c},{ }^{24}$ whereas pure type II behavior is expected at higher temperatures. The experiment in Ref. 6 was done at $T \approx 0.1 T_{c}$ and could thus be repeated at higher temperatures to verify our prediction.

In Fig. 3(a) a similar phase diagram is constructed for recently discovered, and for many reasons exciting, pnictides. In particular, we show the results for LiFeAs, using the parameters given in Ref. 3, except for the fact that $\lambda_{12}$ in the $\Lambda$ matrix must be taken negative due to the $s_{ \pm}$pairing. For this material, we extract $\kappa_{1}=2.4, n_{1} / n_{2}=1.384$ and $v_{1} / v_{2}=0.722$. Interestingly enough, as $\kappa_{2}=\kappa_{1} \sqrt{n_{1} v_{1}^{2} / n_{2} v_{2}^{2}}$, we note that both nominal GL parameters of the bands are larger than $1 / \sqrt{2}$ if $\sqrt{n_{1} v_{1}^{2} / n_{2} v_{2}^{2}} \geq 0.295$. Therefore, it can be once more verified that in a large portion of the parameter space where both bands are convincingly type II, the coupled system exhibits type I behavior. In Fig. 3(b), we show that for $T=0.9 T_{c}$, the $E_{S}=0$ (white) and $\kappa^{*}=1 / \sqrt{2}$ (black) curves coincide for small $n_{1} / n_{2}$ and large $v_{1} / v_{2}$. This behavior persists even at lower temperatures, as shown in Fig. 3(c). However, in the opposite case (large $n_{1} / n_{2}$ and small $v_{1} / v_{2}$ ), the curves separate, forming a region of
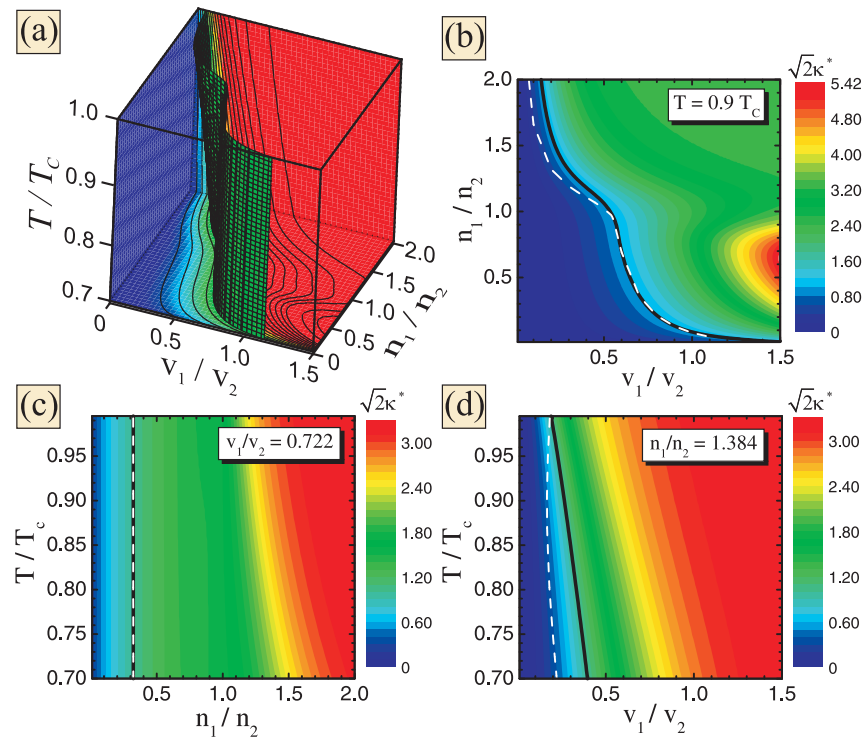

FIG. 3. (Color online) (a) The long-range vortex interaction $\left(v_{1} / v_{2}, n_{1} / n_{2}, T\right)$ phase diagram for LiFeAs for other parameters taken from Ref. 3. The shown isosurface corresponds to $\kappa^{*}=1 / \sqrt{2}$ and the change of the long-range vortex-vortex interaction. (b)-(d) 2D cuts of (a) in the $T=0.9 T_{c}, v_{1} / v_{2}=0.722$, and $n_{1} / n_{2}=1.384$ planes, respectively. Black (white) lines correspond to $\kappa^{*}=1 / \sqrt{2}$ $\left(E_{S}=0\right)$.

nonmonotonic vortex interaction in the phase diagram which grows larger as temperature decreases [see Fig. 3(d)]. This broad temperature range for the observation of partial vortex attraction is important experimentally, to discriminate the nonmonotonic vortex interactions from irregular vortex lattices formed due to intrinsic defects in the material ${ }^{26}$ (with latter being dominant only at temperatures where the vortex core and the defects are similar in size, unless defects are of magnetic nature).

\section{CONCLUSIONS}

In conclusion, we have demonstrated the semianalytic method to relatively easily determine the nature of vortexvortex interaction in two-band superconductors. This is of significant theoretical and experimental importance, as Figs. 2 and 3 sketch just two examples of many possibilities attainable by two-band hybridization. Note that a plethora of transitions, even reentrant behaviors, can be found as a function of the microscopic parameters, which can be tuned experimentally (to some extent) by, for example, carrier injection. ${ }^{27}$ Finally, with appropriate modifications of the initial energy functional our approach can also provide insight in similar situations encountered in nanoscale superconducting films, tailor-made two-component superconducting hybrids, and dirty two-band compounds.

\section{ACKNOWLEDGMENTS}

Discussions with A. Moreira, A. Shanenko, R. Prozorov, and A. Golubov are gratefully acknowledged. This work was supported by the Flemish Science Foundation (FWO-Vl), the Belgian Science Policy (IAP), the bilateral project FWOCNPq, CAPES, and PRONEX/CNPq/FUNCAP. 
*francois.peeters@ua.ac.be

${ }^{1}$ F. Bouquet, R. A. Fisher, N. E. Phillips, D. G. Hinks, and J. D. Jorgensen, Phys. Rev. Lett. 87, 047001 (2001); P. Szabó, P. Samuely, J. Kačmarčík, T. Klein, J. Marcus, D. Fruchart, S. Miraglia, C. Marcenat, and A. G. M. Jansen, ibid. 87, 137005 (2001); M. Iavarone, G. Karapetrov, A. E. Koshelev, W. K. Kwok, G. W. Crabtree, D. G. Hinks, W. N. Kang, E.-Mi Choi, H. J. Kim, H.-J. Kim, and S. I. Lee, ibid. 89, 187002 (2002).

${ }^{2}$ M. L. Teague, G. K. Drayna, G. P. Lockhart, P. Cheng, B. Shen, H.-H. Wen, and N.-C. Yeh, Phys. Rev. Lett. 106, 087004 (2011).

${ }^{3}$ H. Kim, M. A. Tanatar, Y. J. Song, Y. S. Kwon, and R. Prozorov, Phys. Rev. B 83, 100502(R) (2011).

${ }^{4}$ D. Eom, S. Qin, M.-Y. Chou, and C. K. Shih, Phys. Rev. Lett. 96, 027005 (2006); A. A. Shanenko, M. D. Croitoru, A. Vagov, and F. M. Peeters, Phys. Rev. B 82, 104524 (2010); S. Qin, J. Kim, Q. Niu, and C.-K. Shih, Science 324, 1314 (2009); F. M. Peeters, A. A. Shanenko, and M. D. Croitoru, "Nanoscale superconductivity", in Handbook of Nanophysics: Principle and Methods, edited by K. D. Sattler (Taylor and Francis, New York, 2011), Chap. 9.

${ }^{5}$ E. Babaev and M. Speight, Phys. Rev. B 72, 180502 (2005).

${ }^{6}$ V. V. Moshchalkov, M. Menghini, T. Nishio, Q. H. Chen, A. V. Silhanek, V. H. Dao, L. F. Chibotaru, N. D. Zhigadlo, and J. Karpinski, Phys. Rev. Lett. 102, 117001 (2009).

${ }^{7}$ E. H. Brandt and M. P. Das, J. Supercond. Nov. Magn. 24, 57 (2011). ${ }^{8}$ M. Seul and D. Andelman, Science 267, 476 (1995); C. J. Olson Reichhardt, C. Reichhardt, and A. R. Bishop, Phys. Rev. Lett. 92, 016801 (2004).

${ }^{9}$ E. Babaev, J. Carlström, and M. Speight, Phys. Rev. Lett. 105, 067003 (2010).

${ }^{10}$ V. H. Dao, L. F. Chibotaru, T. Nishio, and V. V. Moshchalkov, Phys. Rev. B 83, 020503 (2011).

${ }^{11}$ J. Geyer, R. M. Fernandes, V. G. Kogan, and J. Schmalian, Phys. Rev. B 82, 104521 (2010).

${ }^{12}$ V. G. Kogan and J. Schmalian, Phys. Rev. B 83, 054515 (2011).

${ }^{13}$ V. G. Kogan, C. Martin, and R. Prozorov, Phys. Rev. B 80, 014507 (2009).

${ }^{14}$ M. E. Zhitomirsky and V. H. Dao, Phys. Rev. B 69, 054508 (2004); İ. N. Askerzade, A. Gencer, and N. Güçlü,
Supercond. Sci. Technol. 15, L13 (2002); R. Geurts, M. V. Milošević, and F. M. Peeters, Phys. Rev. B 81, 214514 (2010).

${ }^{15}$ M. R. Eskildsen, M. Kugler, S. Tanaka, J. Jun, S. M. Kazakov, J. Karpinski, and O. Fischer, Phys. Rev. Lett. 89, 187003 (2002); A. E. Koshelev and A. A. Golubov, ibid. 90, 177002 (2003); X. X. Xi, Rep. Prog. Phys. 71, 116501 (2008); M. Silaev and E. Babaev, e-print arXiv:1102.5734v1.

${ }^{16}$ A. A. Shanenko, M. V. Milošević, F. M. Peeters, and A. V. Vagov, Phys. Rev. Lett. 106, 047005 (2011); L. Komendová, M. V. Milošević, A. A. Shanenko, F. M. Peeters, e-print arXiv:1106.1080.

${ }^{17} \mathrm{M}$. Abramowitz and I. A. Stegun, Handbook of Mathematical Functions with Formulas, Graphs, and Mathematical Tables (Dover, New York, 1972), pp. 17-18.

${ }^{18}$ L. Kramer, Phys. Rev. B 3, 3821 (1971)

${ }^{19}$ J. M. Speight, Phys. Rev. D 55, 3830 (1997).

${ }^{20}$ F. Mohamed, M. Troyer, G. Blatter, and I. Luk'yanchuk, Phys. Rev. B 65, 224504 (2002).

${ }^{21}$ A. Gurevich, Physica C 456, 160 (2007).

${ }^{22}$ J.-P. Wang, Phys. Lett. A 374, 58 (2009); Phys. Rev. B 82, 132505 (2010).

${ }^{23}$ A. Chaves, F. M. Peeters, G. A. Farias, and M. V. Milošević, Phys. Rev. B 83, 054516 (2011).

${ }^{24}$ A. A. Golubov, J. Kortus, O. V. Dolgov, O. Jepsen, Y. Kong, O. K. Andersen, B. J. Gibson, K. Ahn, and R. K. Kremer, J. Phys. Condens. Matter 14, 1353 (2002).

${ }^{25}$ This temperature is far out of the GL domain, but is taken as an example. Our prediction can also be made at a higher temperature, but for microscopic parameters that do not correspond to any known material at the moment.

${ }^{26}$ M. R. Eskildsen, L. Ya. Vinnikov, T. D. Blasius, I. S. Veshchunov, T. M. Artemova, J. M. Densmore, C. D. Dewhurst, N. Ni, A. Kreyssig, S. L. Bud'ko, P. C. Canfield, and A. I. Goldman, Phys. Rev. B 79, 100501(R) (2009); L. Ya. Vinnikov, T. M. Artemova, I. S. Veshchunova, N. D. Zhigadlo, J. Karpinski, P. Popovich, D. L. Sund, C. T. Lind, and A. V. Boris, JETP Lett. 90, 299 (2009).

${ }^{27}$ Y. Koval, X. Jin, C. Bergmann, Y. Simsek, L. Özyüzer, Paul Müller, Huabing Wang, G. Behr, and B. Büchner, Appl. Phys. Lett. 96, 082507 (2010) 